\title{
Intratetrad mating, heterozygosity, and the maintenance of deleterious alleles in Microbotryum violaceum (=Ustilago violacea)
}

\author{
MICHAEL E. HOOD \& JANIS ANTONOVICS \\ Department of Biology, University of Virginia, Charlottesville, VA 22903, USA
}

\begin{abstract}
The mating system of Microbotryum violaceum was investigated in populations that are polymorphic for mating-type bias, where individuals produce viable haploids of only one of the two required mating types. The cause of mating-type bias was identified as deleterious recessive alleles linked to mating type. Maintenance of the deleterious alleles was promoted by early conjugation among products of single meioses, such that the duration of the free-living haploid stage is minimized. This development was also observed in nonbiased isolates. As a consequence, the mating system tends toward mating within the tetrad, which might be expected to reduce heterozygosity. However, complete centromere linkage of mating type ensures conjugation between first division meiotic products, such that mating in $M$. violaceum is analogous to forms of meiotic parthenogenesis with first division restitution (i.e. automixis with central fusion). This fungus was used to test the prediction that this mating system would maintain heterozygosity in regions of the genome linked to centromeres. Therefore, populations were screened for additional heterozygous lethal recessive alleles linked to centromeres, and several examples were found. Furthermore, the occurrence of intratetrad mating in $M$. violaceum provides an explanation for low variation among individuals within populations, inconsistent estimates of outcrossing rates, low levels of mating between tetrads of one diploid individual, and high frequencies of haplo-lethal alleles in natural populations.
\end{abstract}

Keywords: automixis, genetic load, heterozygosity, host-pathogen system, mating systems, meiotic parthenogenesis, self fertilization.

\section{Introduction}

In many fungi, the mating-type locus is expressed in the haploid stage as a self-incompatibility mechanism. This has the effect of preventing haploid selfing and immediate homozygosity. However, it may not prevent diploid selfing, which may occur by mating among the products of a single meiosis (automixis) or by mating among the products of different meioses from one diploid individual (autogamy — as found in selfpollinating angiosperms). In fungi, diploid selfing may result from limited dispersal of diploid spores or their meiotic products, and in such cases, it may seem that a mating-type locus would provide no benefit to the maintenance of heterozygosity, except in regions linked to the mating-type locus itself (Mather, 1942). However,

\footnotetext{
*Correspondence. E-mail: michael.hood@virginia.edu
} (C) 2000 The Genetical Society of Great Britain. more recently, it has been pointed out that there is substantial influence of the mating-type locus on the maintenance of heterozygosity depending upon its linkage relationships to the centromere and hence its pattern of segregation (Kirby, 1984; Zakharov, 1986; Merino et al., 1996). If the mating type locus is completely linked to a centromere and undergoes firstdivision segregation, then intratetrad mating will always be between nuclei separated at the first meiotic division (central fusion). Sister nuclei of the second meiotic division are of the same mating type and cannot mate with each other. The important consequence of mating between sister nuclei of the first meiotic division is that heterozygosity will be maintained in regions linked to centromeres. This is because these regions segregate at the first meiotic division and are then restored by the mating process (Lewis \& John, 1963). A breakdown of the haploid self-incompatibility system, random segregation of mating type, or mating between tetrads of one 
diploid individual would disrupt this process and drive centromere-linked regions to homozygosity at a rate of $50 \%$ per mating. In this context, Zakharov (1986) suggested that the high frequency of first division segregation of mating-type loci in fungi with intratetrad mating might be an adaptation to conserve heterozygosity near centromeres throughout the genome.

While there has been conjecture that the relationship between intratetrad mating and the segregation of mating type is important in fungi, there has been no direct demonstration of an effect in the genetics of natural populations. In the present study, we demonstrate the importance of these processes in natural populations of the plant pathogen Microbotryum violaceum, which is the causal organism of anther-smut disease on plants in the Caryophyllaceae.

Mating compatibility in this fungus is controlled by a single mating-type locus with two alleles (Garber \& Day, 1985), and haploid cells must differ at this locus for conjugation to occur. Consequently, this process is often suggested to promote outcrossing (Esser, 1966; Raper, 1966; Elliott, 1994). In particular, M. violaceum has been thought to mate during a free-living haploid generation of yeast-like cells called sporidia (Day, 1979; Antonovics et al., 1996). While this has been frequently posited as the typical life cycle of $M$. violaceum, this view has been brought into question by the presence of biased matingtype ratios in some populations (Kaltz \& Shykoff, 1997; Oudemans et al., 1998). Mating-type bias is characterized by the recovery of haploid sporidia of only one mating type following germination and meiosis of diploid teliospores from one fungal individual. Mating-type bias has also been reported in cereal-smut species (Grasso, 1955; Nielsen, 1968; Darlington \& Kiesling, 1975), which are phylogenetically distant from $M$. violaceum but share a remarkably similar life history (Bauer et al., 1997). It has generally been found that such bias is caused by deleterious alleles linked to the mating-type locus. These alleles are deleterious in the haploid sporidial stage but are recessive and heterozygous in the dikaryotic (or diploid) stage (Fischer \& Holton, 1957; Oudemans et al., 1998). Such alleles have been termed 'haplo-lethals' (Darlington \& Kiesling, 1975) and we continue to use this term for convenience even though, as discussed below, the expression of their deleterious effects may not be invariably lethal.

First, we present direct evidence that haplo-lethal alleles are the cause of mating-type bias in $M$. violaceum. Then, we describe intratetrad mating during postmeiotic development, which serves to explain the persistence of mating-type bias (haplo-lethal alleles) in spite of its apparent disadvantage in the haploid stage. Finally, we report the discovery of heterozygosity in the form of haplo-lethal alleles linked to other centromeres which is predicted by the combination of intratetrad mating and first division segregation of mating type in $M$. violaceum.

\section{The study species}

Microbotryum violaceum (=Ustilago violacea) is a widely distributed fungal pathogen on plants in the Caryophyllaceae. Infected plants are sterilized by altered floral development; the ovaries fail to mature and the fungus develops a dark, powdery spore mass in the anther tissue (Fischer \& Holton, 1957). These teliospores are dispersed when insect pollinators visit flowers on diseased plants (Jennersten, 1983). The teliospores are diploid, and they undergo meiosis during germination to produce a linear tetrad usually consisting of a three-celled promycelium (basidium) plus a cell in the teliospore that is formed when one of the post-meiotic nuclei migrates back into the spore (Hood \& Antonovics, 1998) (Fig. 1a). At the first meiotic division the nuclei in the teliospore cell and the proximal promycelial cell are separated from nuclei in the middle and distal promycelial cells (Fig. 2) (Hood \& Antonovics, 1998). Each cell can then produce haploid sporidia that replicate by yeast-like budding and can be cultured on standard growth media (Hood \& Antonovics, 1998). Mating between haploid cells of opposite mating types (a1 and a2) produces a dikaryotic stage and is a prerequisite for infection (Fischer \& Holton, 1957; Oudemans et al., 1998).

For the current study, host plants of Silene latifolia (= Silene alba) (white campion), with anther-smut disease were collected from a natural population in Virginia (site 6.1-Clover Hollow Rd., Giles Co. VA, USA; Oudemans et al., 1998) and grown under greenhouse conditions. Teliospores from single diseased flowers were stored under desiccation at $25^{\circ} \mathrm{C}$. Each plant was considered to harbour a single genotype of M. violaceum (Baird \& Garber, 1979), and plants were resampled for spores as needed. Additional teliospore collections were made during the 1997 and 1998 annual censuses of this population (Oudemans et al., 1998). Stock cultures of haploid sporidia of known mating types were maintained on potato dextrose agar (PDA) (Difco) at room temperature.

\section{Cause of mating-type bias}

The previous work of Oudemans et al. (1998) suggested that mating-type bias was caused by haplo-lethal alleles linked to mating type, rather than by mechanisms such as spore killers or meiotic drive. Here we report direct observation showing that both mating types are in fact produced, but that one of them is characterized by poor 

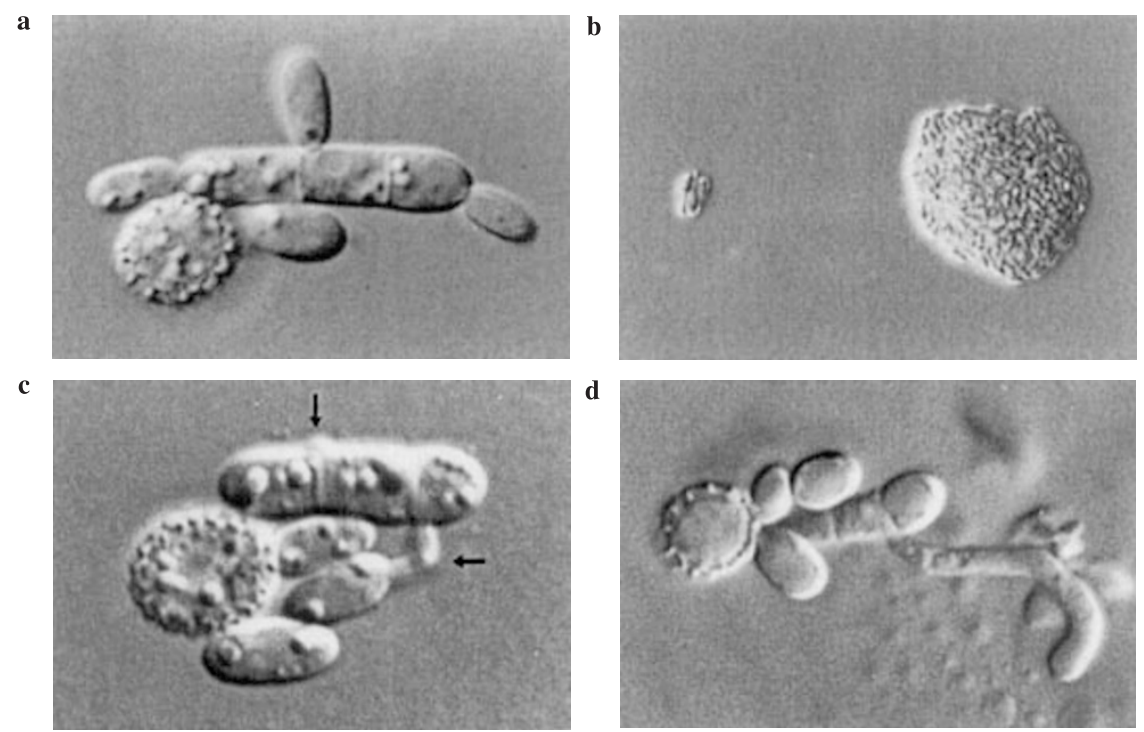

Fig. 1 Post-meiotic development of Microbotryum violaceum. Photographs were taken using differential-interferencecontrast microscopy. (a) Distribution of meiotic tetrad after $24 \mathrm{~h}$ of incubation. Three of the post-meiotic cells are in the promycelium, and the fourth is in the spore wall of the teliospore. Each of these cells has budded one sporidium. The septation between the promycelial cell that is proximal to the teliospore and the middle promycelial cell is formed after the first meiotic division, creating a linear tetrad. (b) Effects of a haplo-lethal allele on sporidial growth after $48 \mathrm{~h}$ of incubation at $25^{\circ} \mathrm{C}$ on $\mathrm{PDA}$. The large colony of sporidia (right) was founded by a viable sporidium, and the small colony (left) was founded by a sporidium from the same tetrad, which contains a lethal allele. (c) Normal pattern of intratetrad conjugations (arrows) in colonies incubated at $15^{\circ} \mathrm{C}$ on water agar. A conjugation is present between the promycelial cell that is proximal to the teliospore cell and the middle promycelial cell. Another conjugation is present between a sporidium produced by the teliospore and the distal promycelial cell. Under these conditions, only the teliospore cell remains unconjugated and able to produce sporidia. (d) Post-meiotic development on the surface of a host plant leading to production of an infection hypha. The conjugation has occurred between two cells of the promycelium (out of focal plane of micrograph), and the sporidia present were most likely produced by the teliospore.

haploid growth. We confirm this by tetrad analysis using micromanipulation.

\section{Methods}

In order to study early sporidial growth in biased and nonbiased isolates, teliospore suspensions from nine isolates from the Clover Hollow population, six biased and three nonbiased, were spread on potato dextrose agar (PDA) and incubated at $25^{\circ} \mathrm{C}$ for $24 \mathrm{~h}$. Preliminary studies showed that each of the promycelial cells and the teliospore had normally budded sporidia after incubation for $24 \mathrm{~h}$ (Fig. 1a), but that these sporidia had generally not undergone a second division. Sporidia within a tetrad were then separated by micromanipulation and transferred singly to another dish of PDA. Growth of each sporidial cell was then followed for several days. An alternative method to study the growth of the meiotic products was to flood the dishes after $24 \mathrm{~h}$ of incubation with $1 \mathrm{~mL}$ of sterile deionized water. The agar surface was scraped, and the resulting suspension was spread on PDA and incubated at $25^{\circ} \mathrm{C}$ and colony growth studied. The presence of haplo-lethal alleles was indicated by large (viable) and small (lethal) sporidial colonies in roughly a 1:1 ratio (Grasso, 1955). Matingtype tests of sporidial cultures were determined by mixing them with sporidia of known mating type and incubating under condition that promote conjugation [at $15^{\circ} \mathrm{C}$ on $1.5 \%$ water agar (water agar; Difco)] (Poon et al., 1974).

\section{Results}

Ordered tetrad analysis of six biased isolates showed complete first division segregation of large and small colonies. For example, the cell in the teliospore wall and the proximal promycelial cell produced large colonies, and the middle and distal promycelial cells produced small colonies, or vice-versa. This result is consistent with linkage to the mating type locus (Hood \& Antonovics, 1998) or another centromere. Mating tests using stock sporidia of known mating type confirmed that sporidia from the large colonies were all of the a1 mating type, indicating the presence of a deleterious allele linked to the a2 mating type. In the small colonies, there were very few sporidia and they appeared 


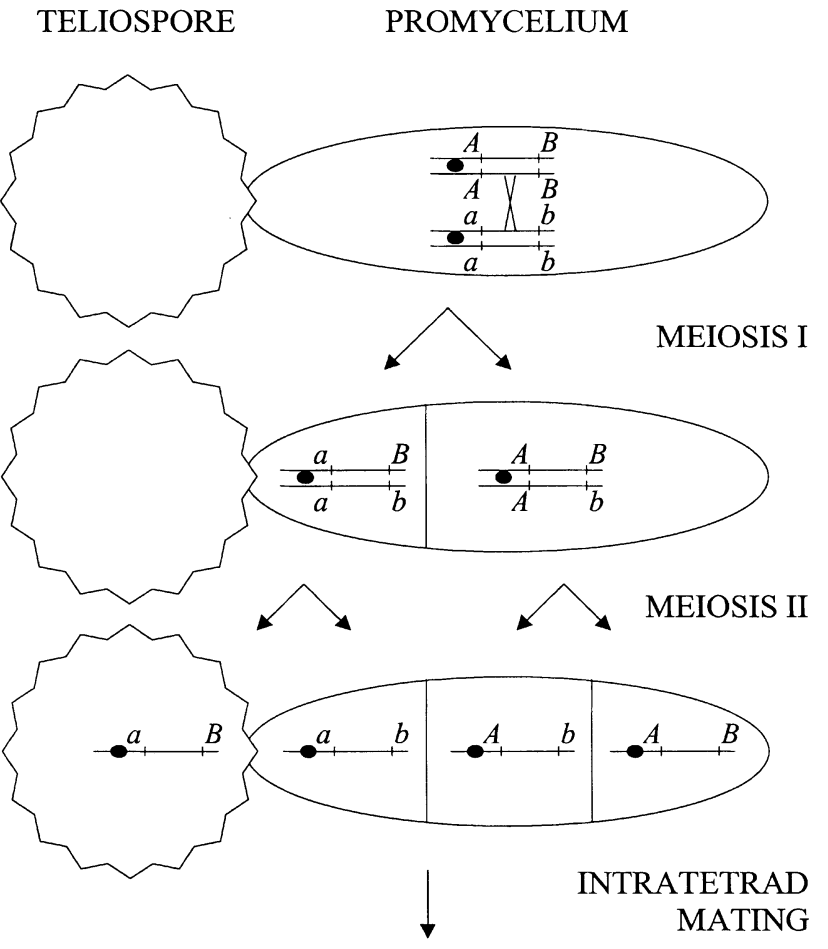

Products of intratetrad mating given first-division segregation of mating type:

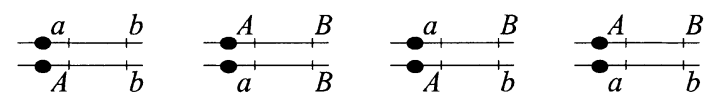

Fig. 2 Diagram of meiosis and intratetrad mating in Microbotryum violaceum. The meiotic tetrad is distributed as three cells of the promycelium and one cell in the teliospore wall. In this model there are two loci (A and B) each of which is heterozygous. The A locus is centromere-linked, while there is one chiasma between the centromere and the B locus. There are four possible products of intratetrad mating with restitution between first division segregants, which is caused by centromere linkage of the mating type locus. All maintain heterozygosity when there is complete linkage to the centromere (i.e. the A locus), but with recombination, two of the products are homozygous (i.e. the B locus).

moribund, vacuolated and with a granular appearance near the cell walls. Sporidia of the large colonies appeared uniformly dense. The three nonbiased isolates produced large colonies from all cells of the tetrad.

The difference in growth rate of a1 vs. a2 sporidia of biased isolates was observed by quantifying replication of cells in the single-sporidial colonies. After $24 \mathrm{~h}$ of incubation, colonies derived from a 1 sporidia contained 9.5 sporidia $(\mathrm{SE}=0.5, n=37)$, and colonies from a2 sporidia contained 2.8 sporidia $(\mathrm{SE}=0.2, n=37$ ). After $48 \mathrm{~h}$ of incubation, the same al colonies contained too many sporidia to count, and the a 2 colonies contained only 3.4 sporidia $(\mathrm{SE}=0.2)$ (Fig. $1 \mathrm{~b})$.

\section{Early meiotic events and intra-tetrad mating}

The presence of alleles that are lethal or highly deleterious in the haploid stage, is at first very puzzling. Previous studies had suggested that their persistence might be the result of avoiding the haploid stage by rapid mating between the meiotic products or by some compensatory advantage in another life history stage (Nielsen, 1968; Antonovics et al., 1998). We hoped to establish whether there were any unusual features of early spore germination and meiosis that would explain the continued transmission and persistence of the haplo-lethal trait. Thus we examined the germination behaviour of biased and nonbiased isolates both in vitro, under a range of temperatures and culture conditions, and in vivo on host surfaces.

\section{Methods}

All experiments were conducted with isolates chosen randomly from those collected in the Clover Hollow population. Two nonbiased isolates (isolates \#58 and $\# 59)$ and two isolates with a1-mating-type bias (isolates \#26 and \#33) were used. Experiments were replicated in four blocks over time except where noted. Regression analyses were conducted using the GLM procedure in SAS (SAS Institute Inc., Cary, NC). Frequency data were arcsin square-root transformed as needed (Steel \& Torrie, 1980).

In order to determine the rate of development during meiosis, the initiation of sporidial production was used as an indicator of completed meiosis. Teliospore suspensions (in sterile deionized water plus a surfactant) were spread on PDA and incubated at $25^{\circ} \mathrm{C}$ for $11 \mathrm{~h}$ $(\approx 500$ spores per $100 \times 15 \mathrm{~mm}$ dish $)$. Among germinated teliospores, the proportion of 100 teliospores and promycelia that had budded at least one sporidium was recorded.

In order to study patterns of conjugation between cells produced in single-teliospore colonies, development was observed under two nutrient treatments (PDA and water agar) and three temperatures $\left(5,15\right.$, and $\left.25^{\circ} \mathrm{C}\right)$. From 12 to $24 \mathrm{~h}$ of incubation, colonies were observed without staining. After $48 \mathrm{~h}$ of incubation, 20 colonies per dish were stained with a small drop of lactophenolcotton blue (Dhingra \& Sinclair, 1985), and the data were recorded as to the presence or absence of each type of conjugation within the colonies. Conjugation types included intra-promycelial, sporidium to promycelium, and sporidium to sporidium.

To investigate whether different types of conjugations produced infection hyphae, we used Phytol solution ( $\alpha$-tocopherol), an agent known to induce hyphal 
formation within the host (Castle \& Day, 1984). Phytol solution was prepared by mixing $6 \mu \mathrm{L}$ of Phytol (Sigma) with $1 \mathrm{~mL}$ of $80 \%$ ethyl alcohol, and then combining $10 \mu \mathrm{L}$ of this solution with $1 \mathrm{~mL}$ of sterile deionized water. Phytol solution $(0.5 \mathrm{~mL})$ was spread on water agar, and the agar was allowed to surface dry under sterile conditions before teliospores were plated. The origin of hyphae in 10 single-teliospore colonies per dish was examined after $24 \mathrm{~h}$ of incubation at $25^{\circ} \mathrm{C}$. This experiment was replicated five times.

The formation of appressoria-like infection structures (Day et al., 1981) was observed under humid conditions on the inner surface of polystyrene Petri dishes. Lids of Petri dishes were sprayed with a mist Phytol solution, dusted with teliospores, and placed back on Petri-dish bottoms containing water agar as a source of humidity. Development of $M$. violaceum was observed after $1-5$ days of incubation at $25^{\circ} \mathrm{C}$.

In order to determine if development in vivo was similar to that observed in vitro, host leaves, flowers, lateral meristems, and cotyledons were inoculated with teliospores. Seeds were obtained from host plants in the Clover Hollow population. Leaves of 1-month-old, greenhouse-grown plants were inoculated by spraying a teliospore suspension onto leaves until runoff $\left(5 \times 10^{6}\right.$ teliospores $/ \mathrm{mL}$ of deionized water plus surfactant). Spray-inoculated plants were incubated in closed chambers of clear plastic that contained wet paper towels to increase humidity. After 5 days at room temperature $\left(22-25^{\circ} \mathrm{C}\right)$, leaves were examined by staining with lactophenol-cotton blue. Floral tissues, including intact flowers and dissected calyx, petal, stamen, and stigma tissues, were inoculated by dusting with teliospores. Inoculated tissues were also incubated in humid chambers at room temperature, and after 3-4 days of incubation, specimens were stained with lactophenolcotton blue. Lateral meristems on bolting stems and in basal rosettes of 2-month-old intact plants were inoculated, incubated, and observed in the same manner as flowers. For inoculation of cotyledons, seeds of $S$. latifolia were surface-sterilized for $5 \mathrm{~min}$ in a solution containing $2 \%$ sodium hypochlorite, $20 \%$ ethyl alcohol, and $0.2 \%$ Triton $\mathrm{X}-100$, and then rinsed in three washes of sterile deionized water. Seeds were germinated on $1.5 \%$ agar containing one-tenth strength Murashige and Skoog Basal Medium (Sigma) and inoculated upon expansion of the cotyledons. Cotyledons were inoculated in the same manner as flowers or by applying a $2 \mu \mathrm{L}$ suspension containing about 2000 teliospores. Specimens were incubated at room temperature for 3-6 days and then observed by staining with lactophenol-cotton blue. All host inoculations were conducted with teliospores of the same nonbiased and biased isolates as used for the in vitro studies. All inoculations for histological investigations were replicated at least four times per isolate.

\section{Results}

Spore germination and post-meiotic development were largely similar between biased and nonbiased isolates, the major difference being somewhat slower development in biased isolates. More germinating teliospores from nonbiased isolates than biased isolates had completed meiosis and initiated budding sporidia after $11 \mathrm{~h}$ of incubation. The proportion of teliospore cells that had budded at least one sporidium was $77 \%(\mathrm{SE}=4.4$, untransformed data, $n=8$ ) for nonbiased isolates, but only $27 \%(\mathrm{SE}=3.3)$ for biased isolates. The proportion of promycelia that had budded at least one sporidium was $55 \%(\mathrm{SE}=4.3)$ for nonbiased isolates, but only $7 \%$ $(\mathrm{SE}=1.8)$ for biased isolates.

The number of different types of conjugation in single-teliospore colonies was significantly influenced by nutrients $(\mathrm{N})$, temperature $(\mathrm{T})$, bias status of isolates (B), and interactions between these treatments (Table 1) (Fig. 3). Three types of conjugations were observed: intra-promycelial (P-P), sporidium-to-promycelium (S-P), and sporidium-to-sporidium ( $\mathrm{S}-\mathrm{S})$. A single colony could contain more than one type of conjugation.

Table 1 Analysis of variance for treatment effects on the proportion of colonies of Microbotryum violaceum with each type of conjugation: intrapromycelial conjugation $(\mathrm{P}-\mathrm{P})$, sporidium-to-promycelium ( $\mathrm{S}-\mathrm{P})$, and sporidiumto-sporidium (S-S). Analysis was conducted using GLM procedures in SAS. Data were arcsine square-root transformed. Note that a single colony may have several types of conjugations simultaneously, and the proportions are therefore calculated as the presence or absence of each conjugation type in 20 single-teliospore colonies (i.e. the proportions of each type are statistically independent). $P$-values represent treatment effects tested against error, except in the cases of the effect of bias and its interactions which were tested against the Type III MS of isolates within those treatments. There were not significant differences between isolates within biased and nonbiased classes

\begin{tabular}{lrccc}
\hline Treatments & d.f & P-P & S-P & S-S \\
\hline Block & 3 & NS & NS & NS \\
Nutrient & 1 & $* *$ & $* *$ & $* *$ \\
Temperature & 2 & $* *$ & $* *$ & $* *$ \\
Bias & 1 & $*$ & NS & $* *$ \\
$\mathrm{~N} \times \mathrm{T}$ & 2 & $* *$ & $* *$ & $* *$ \\
$\mathrm{~N} \times \mathrm{B}$ & 1 & $* *$ & + & $*$ \\
$\mathrm{~T} \times \mathrm{B}$ & 2 & $*$ & $\mathrm{NS}$ & $*$ \\
$\mathrm{~N} \times \mathrm{T} \times \mathrm{B}$ & 2 & $\mathrm{NS}$ & $\mathrm{NS}$ & $* *$ \\
Error & 69 & & & \\
\hline
\end{tabular}

${ }^{* *} P<0.01 ; * P<0.05 ;{ }^{+} P<0.1$; NS, nonsignificant. 

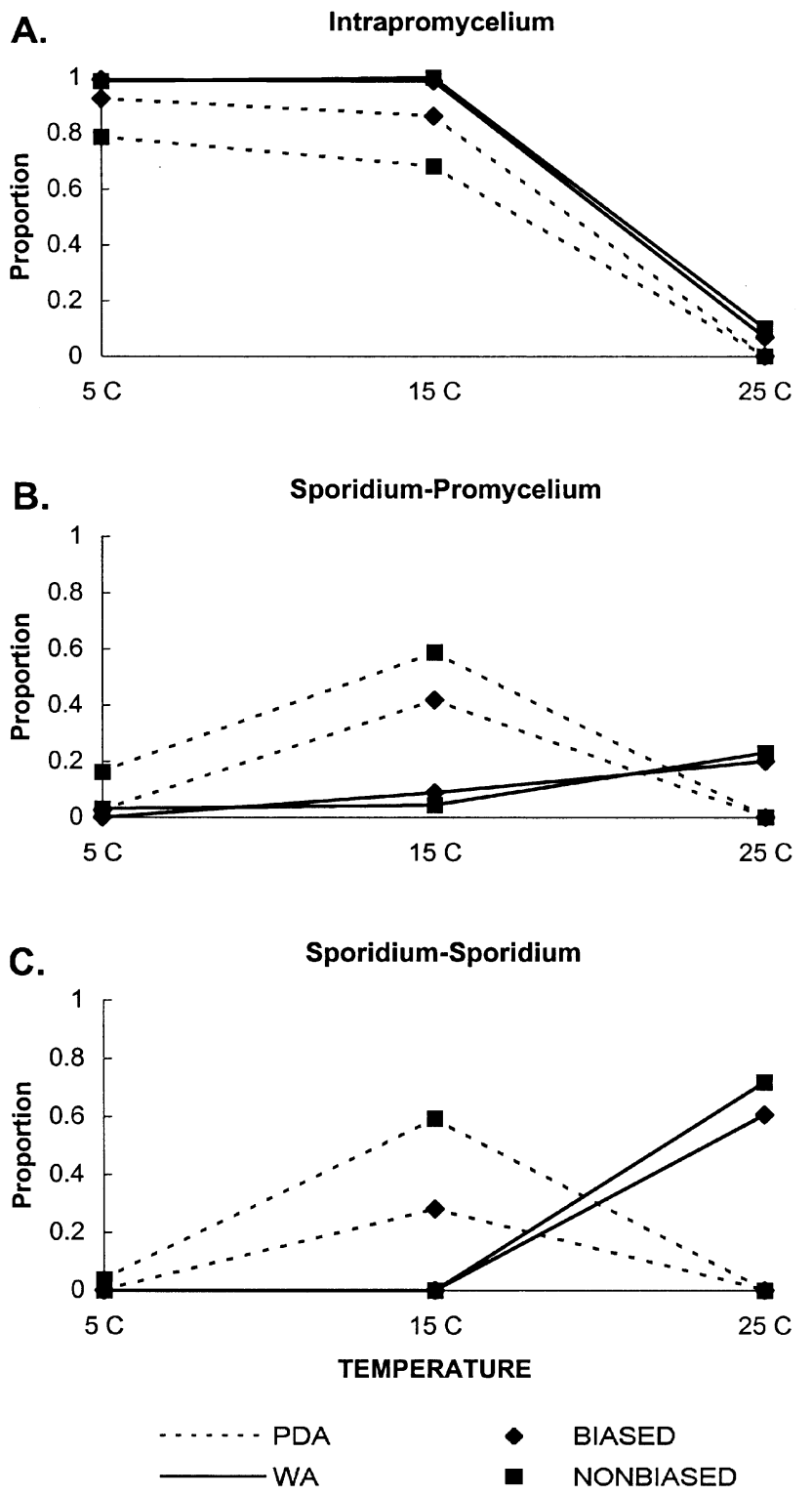

Fig. 3 Proportion of single teliospore colonies of Microbotryum violaceum with each type of conjugation under nutrient and temperature treatments. A single colony could contain more than one type of conjugation. Biased and nonbiased isolates were compared on media of two nutrient levels: potato dextrose agar (PDA) and water agar. Two biased and two nonbiased isolates were used in the experiment, with 180 observations per treatment, but data were combined for presentation within the biased and nonbiased classifications (Duncan's Multiple Range test). Comparison of bias effects across treatment combinations were determined using the LSMEANS procedures in SAS. Biased and nonbiased isolates differed on PDA at $15^{\circ} \mathrm{C}$ for each type of conjugation (largest $P=0.0004)$, and on PDA at $5^{\circ} \mathrm{C}$ they differed for intra-promycelial $(P=0.0003)$ and sporidia-to-promycelia conjugations $(P=0.0034)$. On water agar, biased and nonbiased isolates differed only for sporidia-to-sporidia conjugations at $25^{\circ} \mathrm{C}(P=0.0102)$.
There is an expected negative correlation between $\mathrm{P}-\mathrm{P}$ conjugations and conjugations involving sporidia. This negative correlation is because $\mathrm{P}-\mathrm{P}$ conjugations can occur so rapidly as to preclude the production of sporidia from promycelial cells (Hood \& Antonovics, 1998). Intra-promycelial conjugations dominated at lower temperatures and nutrients, with conjugations involving sporidia becoming frequent only at $15^{\circ} \mathrm{C}$ on PDA and at $25^{\circ} \mathrm{C}$ on water agar. No conjugations of any type were observed at $25^{\circ} \mathrm{C}$ on PDA.

A typical pattern of intratetrad conjugations on water agar at $15^{\circ} \mathrm{C}$ included an intra-promycelial conjugation between the proximal promycelial cell and the middle cell, and a conjugation between a sporidium produced by the teliospore cell and the distal promycelial cell (Fig. 1c). This pattern of conjugation is consistent with first division segregation of mating type (Fig. 2).

Hyphae produced in single-teliospore colonies most often originated from conjugations involving promycelial cells. Biased and nonbiased isolates did not differ in this behaviour. Among the 200 single-teliospore colonies examined, 132 produced one hypha and 54 produced two hyphae. Among these 240 hyphae, 175 originated from intrapromycelial conjugations and 55 originated from conjugations between a promycelial cell and a sporidium. Only 10 originated from conjugations between two sporidia. Among the 54 colonies producing two hyphae, 48 colonies produced one hypha originating from an intra-promycelial conjugation and one from a conjugation between a promycelial cell and a sporidium. This development corresponds to the two types of conjugations typical of colonies at $15^{\circ} \mathrm{C}$ on water agar. The other six colonies with two hyphae contained one hypha originating from a conjugation involving a promycelial cell and one hypha originating from a sporidium-sporidium conjugation.

Microbotryum violaceum formed appressoria-like infection structures, similar to those reported by Day et al. (1981), on the hydrophobic surface of polystyrene Petri dishes under humid conditions after 3 days of incubation. Staining of cytoplasm was often restricted to the swollen apex and a short distance back into the hypha. Development did not differ between biased and nonbiased isolates.

Development on host tissue was similar to that observed in vitro. Biased and nonbiased isolates did not differ in the production of hyphae or infection structures on host tissues. Teliospores germinated and produced promycelia which were two- or three-celled: the development of two-celled promycelia is described by Hood \& Antonovics (1998). Intrapromycelial conjugation was particularly common, but sporidium-to-promycelium and sporidium-to-sporidium conjugations also were 
observed. Hyphae produced on host tissue were only observed originating from conjugations involving promycelial cells (Fig. 1d). More than 50 events of hyphal production by each isolate were observed on each type of host tissue (leaf tissue, floral tissue, lateral meristems, and cotyledons). In every case, the hyphae originated from either intrapromycelial conjugations or conjugations between promycelial cells and sporidia. Hyphae were often short $(20-40 \mu \mathrm{m})$ and unbranched, but where the inoculation site remained wet during incubation (i.e. some inoculated cotyledons), hyphae were much longer. Conjugated cells that produced hyphae were often devoid of cytoplasm, staining being restricted to the distal end $(12.0 \mu \mathrm{m}, n=20, \mathrm{SE}=1.2)$ of the undifferentiated hyphal apex. Some hyphal apices on host tissue were swollen and resembled appressoria. Infection structures were most readily observed on lateral meristems, cotyledons, and petal and calyx tissues.

\section{Centromere linkage of haplo-lethal alleles}

Given that there is complete first division segregation of mating type (Hood \& Antonovics, 1998) and intratetrad mating, the mating system in $M$. violaceum is analogous to other automictic mating systems, including forms of meiotic parthenogenesis with restitution between products of the first meiotic division. A number of workers have pointed out, in the context of meiotic parthenogenesis (Lewis \& John, 1963) as well as fungal mating systems (Zakharov, 1986), that this form of mating can be a mechanism for maintaining heterozygosity in all regions of the genome linked to centromeres. We therefore set out to test the prediction that haplo-lethal alleles may be found not only linked to mating type, but also at other centromeres. Below we describe methods developed to test this prediction.

\section{Methods}

Conjugations between sporidia were usually absent in single-teliospore colonies at low temperatures and nutrients (Fig. 3c) because all the promycelial cells conjugated so rapidly that they were unable to produce any sporidia. The proximal and middle promycelial cells conjugated with each other, and the distal promycelial cell conjugated with a sporidium budded from the teliospore cell. Therefore, only the haploid cell within the wall of the teliospore remained unconjugated and continued to bud sporidia, resulting in a sporidial colony of a single haploid genotype and thus a single mating type (Fig. 1c). Given a heterozygous locus for a haplo-lethal allele, the probability of the lethal allele segregating into this teliospore nucleus is 0.5 . This was confirmed by incubating teliospores of biased isolates on water agar at three temperatures $\left(5,15\right.$, and $\left.25^{\circ} \mathrm{C}\right)$ for 7 days. At 15 or $5^{\circ} \mathrm{C}$ on water agar, half $(49 \%, n=600)$ of single-teliospore colonies failed to grow beyond 4-6 sporidia, suggesting that they were founded by sporidia of the lethal a2 mating type; the remaining colonies grew to contain many sporidia. Therefore, the first test was for the presence of a haplo-lethal allele. Teliospores were incubated at $15^{\circ} \mathrm{C}$ on water agar and examined for a $1: 1$ ratio of small to large colonies.

Where haplo-lethal alleles were detected, the linkage relationship of the haplo-lethal alleles to mating type was then assessed as follows. If all large colonies at $15^{\circ} \mathrm{C}$ on water agar were of a single mating type (eight to 10 colonies tested per isolate), then the haplo-lethal allele was mating-type linked. Otherwise, if the mating type varied between large colonies, the linkage of the haplo-lethal allele to the centromere of another chromosome was determined by the following method. Teliospores were incubated at $25^{\circ} \mathrm{C}$ on PDA, so that all cells of the tetrad would be represented by sporidia. The mating type was determined in 10 teliospore colonies per isolate. Centromere linkage (to a nonmating-type centromere) was indicated if each teliospore colony contained sporidia of only a single mating type, but the mating types varied at a 1:1 ratio among colonies. Mating type was determined by methods described above.

\section{Results}

A total of 149 samples from the Clover Hollow population were tested using the methods described above. Haplo-lethal alleles were detected in 116 of these isolates by the production of small and large colonies in a $1: 1$ ratio on water agar at $15^{\circ} \mathrm{C}$. A lethal allele was linked to the a 2 mating type in 110 of these isolates, indicated by only a1-containing large colonies. Two of the remaining six isolates contained haplo-lethal alleles linked to another (nonmating type) centromere. This result was indicated by the presence of teliospore colonies, all of which contained only one mating type at $25^{\circ} \mathrm{C}$ on PDA, but where the ratio of mating types among colonies was 1:1. For example, of 106 singleteliospore colonies from isolate \#41,51 contained only a1 sporidia, 52 contained only a2 sporidia, and 3 contained sporidia of both mating types. The latter class may represent rare recombinants, but further studies are needed to confirm this. The source plant of one of these isolates was sampled during the 1996 census, and testing of the stored teliospores confirmed that the centromerelinked allele was present in that year as well.

The four remaining isolates provided evidence of multiple haplo-lethal alleles within single isolates. This was indicated by ratios of small to large colonies at $15^{\circ} \mathrm{C}$ 
on water agar that differed from the predicted 1:1 ratio for a single haplo-lethal allele. If two unlinked haplolethal alleles are present, the probability of the teliospore cell not receiving either during meiosis is 0.25 , and therefore the predicted ratio of large to small colonies is 1:3. Three of the isolates gave such a ratio: (large:small colonies) isolate \#75, 25:79; isolate \#76, 39:97; isolate $\# 99,41: 106$. The following lines of evidence were used to establish that one of the haplo-lethal alleles was linked to mating type and the other was linked to another centromere. Linkage of one haplo-lethal allele to the mating type locus was inferred because the sporidia of large colonies were all of a 1 mating type. Linkage of the second haplo-lethal to a nonmating-type centromere was inferred because, when each cell of the tetrad produced sporidia (at $25^{\circ} \mathrm{C}$ on PDA), only half of the single-teliospore colonies were large: (large:small colonies) isolate \#75, 214:286; isolate \#76, 198:302; isolate \#99, 237:263. This is because the probability of two centromere-linked haplo-lethal alleles cosegregating into one daughter nucleus of the first meiotic division is 0.5 , and these meioses would produce viable sporidia only from two cells of the tetrad. Likewise, the probability of the alleles segregating to opposite nuclei at the first meiotic division is also 0.5 , and all tetrad cells of these meioses would contain a lethal allele and would produce no viable sporidia. In contrast, with one centromere linked and one centromere unlinked haplo-lethal allele (undergoing second division segregation at a rate of 0.5 ) the expected ratio of large to small colonies at $25^{\circ} \mathrm{C}$ on PDA would be 1:3. Ratio values between 1:1 and 1:3 may suggest less than complete linkage of the second haplo-lethal allele to the centromere. The remaining isolate provided a large:small colony ratio of 7:106 at $15^{\circ} \mathrm{C}$ on water agar, where the large colonies were all of a1 mating type. This suggests the presence of more than two haplo-lethal alleles in this isolate. Additional studies of isolates with more than one haplo-lethal allele are required for analysis of precise linkage relationships and recombination frequencies.

\section{Discussion}

The occurrence of intratetrad mating implies that the free-living haploid stage is bypassed, and this presents an opportunity for the persistence of mutations that are harmful in the haploid stage, but which are recessive and therefore have no deleterious effect in the diploid stage. If intratetrad mating occurs at a very high frequency, then such deleterious recessive mutations may persist in association with nonrecombining regions, similar to what might be expected in other regions of the genome that are maintained as permanent heterozygotes (e.g. structural heterozygosity as in Oenothera, alleles on heterogametic sex-chromosomes, alleles associated with self-incompatibility loci in diploids). The frequency of such mutations may even be enhanced by the presence of transposable elements in these regions, as has been suggested in Microbotryum (Garber \& Ruddat, 1994). Alternatively, the haploid stage may not be circumvented altogether, and the persistence of haplo-lethals may be because they have compensatory advantageous effects in the diploid phase (Darlington \& Kiesling, 1975; Oudemans et al., 1998). We have shown theoretically that haplo-lethal alleles can spread to high frequency, depending on their advantage in the diploid phase and the degree of intratetrad mating (Antonovics et al., 1998). Moreover, populations with frequencies of mating-type bias approaching $100 \%$ have been observed in nature (Oudemans et al., 1998).

Our observations indicate that intratetrad mating is likely to be a consistent feature of the life-history of $M$. violaceum. The evidence for this is both direct, in the form of observation of development in teliospore colonies, as well as indirect, in that this phenomenon helps explain the persistence of mating-type linked haplo-lethal alleles and hence the common occurrence of mating-type bias in natural populations. As the duration of the haploid stage is reduced, the likelihood of conjugations occurring among replicating sporidia in a free-living haploid generation is also reduced. Instead, the types of conjugations that predominate would be those that involve the cells first available within the tetrad. In fact, cells of the promycelium and the initially produced sporidium from the post-meiotic teliospore are involved in the great majority of conjugations under the low nutrient regimes and at temperatures that are optimal for infection. These conjugations are by far the most common source of the infection hyphae in vitro and on the surface of host plants. As a result, the mating system would tend toward mating within the tetrad, yet where this process is still governed by the bipolar incompatibility system at the mating type locus.

There is a direct parallel between the mating system of $M$. violaceum and some forms of meiotic parthenogenesis. Specifically, parthenogenetic automixis involves meiotic divisions, and then restitution or fusion of the products to restore the diploid condition without genetic contribution from another individual. Varied and complicated mechanisms are often involved in restitution, but a common feature of most systems is the conservation of heterozygosity in certain parts of the genome (Lewis \& John, 1963). For example, in meiotic parthenogenesis in moths, where females are the heterogametic sex, there is also first division restitution of meiotic products and complete suppression of crossing over (Suomalainen et al., 1976), which ensures the maintenance of heterogamety in females. The observation of 
haplo-lethal alleles at additional centromeres in $M$. violaceum is consistent with the expectation that heterozygosity will be maintained in centromeric regions by the combination of intratetrad mating and the first division segregation of the mating type. In addition, colony colour is frequently heterozygous in natural populations of $M$. violaceum, and is completely centromere linked but not linked to mating type (Garber et al., 1978; Garber \& Day, 1985).

The technical difficulty of directly observing the mating systems of plants and fungi in nature has led to the common use of genetic markers to assess mating systems (Ross, 1986; Milgroom et al., 1993; Gitzendanner et al., 1996). However, because intratetrad mating combined with first division segregation of mating type in $M$. violaceum may maintain heterozygosity linked to centromeres, the use of genetic markers without prior knowledge of their linkage relationships is potentially very misleading. A similar point has been made in the context of homothallic mushrooms (Kerrigan, 1990). Earlier, unpublished studies by P.V. Oudemans and H.M. Alexander (pers. com. 1997), using mating-type unlinked RAPD markers, gave estimates of outcrossing rates that seemed unrealistically high $(40-60 \%)$. These estimates also differed from the low rates (6.6\%) observed when isolates containing contrasting genotypes were deliberately used in mixed teliospore inoculations (Baird \& Garber, 1979). While Oudemans and Alexander questioned their results on the basis of the reliability of the RAPD markers they used, the high outcrossing rates which they obtained may have been because the markers, while unlinked to mating type, were linked to other centromeres. Garber et al. (1987) reported significant suppression of recombination near centromeres in $M$. violaceum, with few loci beyond $10 \mathrm{cM}$ from the centromeres. This would further increase the chances of choosing markers inappropriate for estimating the mating system.

Once the combination of mating within the tetrad and first division segregation of mating type is established, accumulation of deleterious recessive mutations at each centromere would further restrict the organism from other forms of mating. As mentioned above, only mating within tetrads will maintain heterozygosity in these regions, while mating between tetrads would result in $50 \%$ of centromeres becoming homozygous (except the centromere containing the mating type locus). In doing so, deleterious recessive mutations at centromeres would be exposed and reduce the fitness of products resulting from mating between tetrads. Support for this hypothesis comes from the studies of Baird \& Garber (1979), who found evidence of far less mating between tetrads than would be expected by chance alone; only 2 of 78 infections provided evidence of mating between tetrads using concentrated suspensions of teliospores of single genetically marked isolates as inoculum. With intratetrad mating reinforced, we can envisage that populations of $M$. violaceum may consist of a limited number of genetically similar lines. This is because intratetrad mating will preserve heterozygosity linked to centromeres and will rapidly drive other regions of the genome to homozygosity. Therefore, mating within the tetrad will regenerate the diploid parental genotype once heterozygous loci are eliminated from regions not linked to centromeres. This prediction is supported by the recent study of Delmotte et al. (1999), who found that there was relatively little within-population variation for microsatellite markers but a large amount of variation between populations in Europe ( $F_{\mathrm{ST}}$ of 0.904$)$. This is also consistent with general uniformity in traits within and between populations, such as electrophoretic markers (Antonovics et al., 1996) and virulence (Alexander et al., 1993) previously reported within populations of this species.

The precise role of the sporidial stage in the life history of this fungus remains unclear. In the laboratory, most observations on the early growth and germination of teliospores have been carried out on media containing high nutrients and at room temperature (Poon et al., 1974), and under these conditions sporidia do not conjugate, but continue to bud asexually. It is only under conditions of nutrient starvation (e.g. by transfer to water agar) that the sporidia can be induced to conjugate, and such conjugating sporidia are commonly used for artificial inoculation (Garber \& Day, 1985; Antonovics et al., 1996).

We suspect that $M$. violaceum in reality has a mating system that is a mixture of intratetrad mating and rare outcrossing. However, the relative frequencies of these two types of events in nature remain unknown. The average amount of heterozygosity that exists within individuals will depend largely upon the frequency of intratetrad mating vs. outcrossing and the relatedness between individuals if outcrossing occurs (as well as, of course, the rate of mutation, and the frequency of recombination). It is possible that sporidia production is a byproduct of efficient intratetrad mating as sporidia production by the teliospore is necessary for conjugation between the distal cell of the promycelium and the haploid nucleus in the teliospore. Using genetic markers with known centromere linkage relationships in experimental populations should make it possible to estimate the relative frequencies of intratetrad mating, mating between tetrads, and outcrossing.

The experiments reported here also show that the early post-meiotic development, which leads to intratetrad mating, occurs in both biased and nonbiased fungal genotypes. The occurrence of intratetrad mating 
thus appears to be a regular feature of normal genotypes and not in and of itself caused by the presence of a haplo-lethal allele. However, it currently remains an open question as to whether the somewhat greater intratetrad mating in biased isolates is the result of early expression of the haplo-lethal allele in the promycelium (i.e. a pleiotropic effect of some metabolic deficiency, which slows sporidia formation and promotes early conjugation of promycelial cells), or if, alternatively, the slightly greater rate of intratetrad mating in biased isolates is the result of selection that has increased this rate. Further genetic studies are needed to establish the genetic basis of intratetrad mating, and to examine whether its frequency is higher in populations where haplo-lethals are common.

The whole 'syndrome' of intratetrad mating appears to have been evolved a number times in the fungi (or has been retained as an ancestral state). It is another example of the remarkable similarities in the reproductive biology of the dicotyledonous smuts, such as $M$. violaceum, and the grass smuts. While these two groups were formerly considered to belonging in the same genus, it is now clear (Bauer et al., 1997) that the group to which $M$. violaceum belongs (Microbotryales) is phylogenetically more closely related to the rusts (Uredinales), and is as distantly related to the grass smuts (Ustilaginales) as to the mushrooms (Agaricales). Mating-type bias caused by haplo-lethal alleles is also common to both groups of smut fungi, and therefore appears to be a predictable consequence of this mating system (Grasso, 1955; Nielsen, 1968; Darlington \& Kiesling, 1975).

Numerous species of ascomycete fungi also exhibit intratetrad mating in the form of pseudo- or secondaryhomothallism. In these fungi, meiosis is followed by a post-meiotic mitosis, and then nuclei of opposite mating type are packaged into self-fertile dikaryotic ascospores. Many genetic consequences of intratetrad mating in $M$. violaceum are similar to the predictions by Merino et al. (1996) regarding the secondary homothallic ascomycete, Neurospora tetrasperma. These include the predicted maintenance of heterozygosity linked to centromeres and the accumulation of 'nonfunctional alleles'. Intra-tetrad mating is also common is some yeasts, such as Saccharomycodes ludwgii and Saccharomyces cerevisiae (Zakharov, 1987).

Our studies show that simple knowledge of the genetics of the incompatibility system of fungi is inadequate to predict both the mating system as well as how the genetic variation is structured at the level of the genome and the population. Thus we have shown that in $M$. violaceum, while there is a bipolar selfincompatibility system, the predominant mode of reproduction is most likely via intratetrad mating.
Furthermore, while such selfing may seem at first sight to be the most extreme possible, nonetheless in conjunction with first division segregation of the mating type, it may lead to the preservation of heterozygosity. While heterozygosity is preserved within-individuals, there is low genetic variation among individuals within the population as a whole. Therefore, there is not the positive correlation between the amount of genetic variation at the genome level and at the population level that is expected for sexual organisms (Lewis \& John, 1963). In addition to contributing to our understanding of the coevolutionary dynamics of the Microbotryum-Silene host-pathogen systems, our studies show that $M$. violaceum is a useful model of meiotic parthenogenesis and automixis, in that predictions about genome structure can be tested using detailed tetrad analysis.

\section{References}

ALEXANDER, H. M., ANTONOVICS, J., AND KELLY, A. W. 1993. Genotypic variation in plant disease resistance - physiological resistance in relation to field disease transmission. J. Ecol., 81, 325-333.

ANTONOVICS, J., STRATTON, D., THRALL, P. H. AND JAROSZ, A. M. 1996. An anther-smut disease (Ustilago violacea) of fire-pink (Silene virginica): its biology and relationship to the anthersmut disease of white campion (Silene alba). Am. Midl. Nat., 135, 130-143.

ANTONOVICS, J. O., KEEFE, K. AND HOOD, M. E. 1998. Theoretical population genetics of mating-type linked haplo-lethal alleles. Int. J. Plant Sci., 159, 192-198.

BAIRD, M. L. AND GARBER, E. D. 1979. Genetics of Ustilago violacea. V. Outcrossing and selfing in teliospore inocula. Bot. Gaz., 140, 89-93.

BAUER, R., OBERWINKLER, F. AND VANKY, K. 1997. Ultrastructural markers and systematics in smut fungi and allied taxa. Can. J. Bot., 75, 1273-1314.

CASTLE, A. J. AND DAY, A. 1984. Isolation and identification of $\alpha$-tocopherol as an inducer of the parasitic phase of Ustilago violacea. Phytopathology, 74, 1194-1200.

DARLINGTON, L. C. AND KIESLING, R. L. 1975. A compatibilitylinked, haplo-lethal factor in race 1, Ustilago nigra (Tapke). North Dakota Acad. Sci., 27, 116-119.

DAY, A. W. 1979. Mating type and morphogenesis in Ustilago violacea. Bot. Gaz., 140, 94-101.

DAY, A. W., CASTLE, A. J. AND CUMmins, J. E. 1981. Regulation of parasitic development of the smut fungus, Ustilago violacea, by extracts from host plants. Bot. Gaz., 142, 135-146.

Delmotte, F., BuCheli, E. AND ShykofF, J. A. 1999. Host and parasite population structure in a natural plant-pathogen system. Heredity, 82, 300-308.

DHINGRA, O. D. AND SINCLAIR, J. B. 1985. Basic Plant Pathology Methods, 2nd edn. CRC Press, Boca Raton, FL.

ELliotT, G. C. 1994. Reproduction in Fungi. Chapman \& Hall, London.

(c) The Genetical Society of Great Britain, Heredity, 85, 231-241. 
ESSER, K. 1966. Incompatibility. In: The Fungi. An Advanced Treatise, pp. 661-678. Academic Press, New York.

Fischer, G. W. AND HOLTON, C. S. 1957. Biology and Control. of the Smut Fungi. Ronald Press, New York.

GARBER, E. D. AND DAY, A. W. 1985. Genetic mapping of a phytopathogenic basidiomycete, Ustilago violacea. Bot. Gaz., 146, 449-459.

GARBER, E. D. AND RUDDAT, M. 1994. Genetics of Ustilago violacea. XXXII. Genetic evidence for transposable elements. Theor. Appl. Genet., 89, 838-846.

GARBER, E. D., BAIRD, M. L. AND WEISS, L. M. 1978. Genetics of Ustilago violacea. II. Polymorphism of colony color and nutritional requirements of sporidia from natural populations. Bot. Gaz., 139, 261-265.

GARBER, E. D., ENG, C. H. AND STEVENS, D. M. 1987. Genetics of Ustilago violacea. XXI. Centromere-linkage values and pericentric gene clustering. Curr. Genet., 12, 555-560.

GITZENDANNER, M. A., WHITE, E. E., FORD, B. M., DUPPER, G. E., HODGSKISS, P. D. AND KINLOCK, B. B. 1996. Genetics of Cronartium ribicola. III. Mating system. Can. J. Bot., 74, 1852-1859.

GRASSO, v. 1955. A haplo-lethal deficiency in Ustilago kolleri. Phytopathology, 45, 521-522.

HOOD, M. E. AND ANTONOVICS, J. 1998. Two-celled promycelia and mating-type segregation in Ustilago violacea ( $=$ Microbotryum violaceum). Int. J. Plant Sci., 159, 199-205.

JENNERSTEN, O. 1983. Butterfly visitors as vectors of Ustilago violacea spores between caryophyllaceous plants. Oikos, 40, $125-130$.

KALTZ, O. AND SHYKOFF, J. A. 1997. Sporidial mating type ratios of teliospores from natural populations of the anther smut fungus, Microbotryum (=Ustilago) violaceum. Int. J. Plant Sci., 158, 575-584.

KERRIGAN, R. W. 1990. Evidence of genetic divergence in two populations of Agaricus bisporus. Mycol. Res., 94, $721-733$.

KIRBY, G. C. 1984. Breeding systems and heterozygosity in populations of tetrad-forming fungi. Heredity, 52, 35-41.
LEWIS, K. R. AND JOHN, B. 1963. Chromosome Marker. Little and Brown, Boston, MA.

MATHER, K. 1942. Heterothally as an outbreeding mechanism in fungi. Nature, 142, 54-56.

MERINO, S. T., NELSON, M. A., JACOBSON, D. J. AND NATVIG, D. O. 1996. Pseudohomothallism and evolution of the mating-type chromosome in Neurospora tetrasperma. Genetics, 143, 789-799.

MILGROOM, M. G., LIPARI, S. E., ENNOS, S. E. AND LIU, Y. C. 1993. Estimation of the outcrossing rate in the chestnut blight fungus, Cryphonectria parasitica. Heredity, 70, 385-392.

NIELSEN, J. 1968. Isolation and culture of monokaryotic haplonts of Ustilago nuda, the role of proline in their metabolism, and the inoculation of barley with resynthesized dikaryons. Can. J. Bot., 46, 1193-1200.

OUdEMANS, P. V., ANTONOVICS, J., ALTIZER, S. M., THRALl, P. H., ROSE, L. AND ALEXANDER, H. M. 1998. The distribution of mating-type bias in natural populations of the anther smut Ustilago violacea on Silene alba in Virginia. Mycologia, 90, 372-381.

POON, N., MARTIN, J. AND DAY, A. W. 1974. Conjugation in Ustilago violacea. I. Morphology. Can. J. Microbiol., 20, 187-191.

RAPER, J. R. 1966. Life cycles, basic patterns of sexuality, and sexual mechanisms. In: The Fungi. An Advanced Treatise, pp. 473-512. Academic Press, New York.

Ross, H. A. 1986. Estimation of mating system parameters in plant populations using marker loci with null alleles. Theor. Appl. Genet., 72, 322-327.

STEEL, R. G. D. AND TORRIE, J. H. 1980. Principles and Proceedings of Statistics: A Biometrical Approach, 2nd edn. McGrawHill, New York.

SUOMALAINEN, E., SAURA, A. AND LOKKI, J. 1976. Evolution of parthenogenetic insects. Evol Biol., 9, 209-257.

ZAKHAROV, I. A. 1987. Some principles of the gene localization in eukaryotic chromosomes. Formation of the problem and analysis of nonrandom localization of the mating-type loci in some fungi. Genetika, 22, 2620-2624. 